\title{
Test anxiety, input modality, and levels of organization in free recall
}

\author{
JOHN H. MUELLER \\ University of Missouri, Columbia, Missouri 65201
}

\begin{abstract}
Subjects performed for five trials on a free recall list containing both rhyming word pairs and word-associate pairs, with either visual or auditory presentation. High-anxiety subjects recalled and clustered less than low-anxiety subjects for both rhymes and associates. There was little effect due to input modality, nor did modality interact with anxiety.
\end{abstract}

Recent research has shown that high-anxiety subjects do not perform as well as low-anxiety subjects in free recall (cf. Mueller, 1976). Specifically, although highanxiety subjects may recall as many words, their recall is less organized; for example, there is less clustering of words in terms of intralist relationships such as taxonomic category membership. One interpretation of this deficit is that high-anxiety subjects engage in a more restricted encoding of the items, and thus are less able to utilize various word features to advantage in organizing material in memory.

A question of interest concerns the generality of this cue utilization deficit. It has been observed (Broadbent, 1971; Schwartz, 1975) that states of high arousal seem to orient the subject's attention to the physical characteristics of linguistic materials, at the expense of semantic content. One way to conceptualize this is in terms of the depth-of-processing analysis (Craik \& Lockhart, 1972). This view assumes that "shallow" processing of a word involves attending to physical features, that is, its orthography or sound, with the result being an unstable or transitory memory trace. "Deep" processing is said to occur when semantic content is examined, and it results in a stronger memory trace. One hypothesis, then, might be that high-anxiety subjects have a deficit in processing deep (semantic) features, but no deficit in terms of processing shallow (physical) attributes. While arousal and anxiety may not be identical, the arousal results noted above are consistent with this interpretation.

A test of this hypothesis should be possible in free recall, given that the word list involves both shallow and deep dimensions for organization. The present experiment used a list construction with both rhyming (shallow) and word-associate (deep) relationships in the same list (cf. Dolinsky, 1972). With such a list, subjects can cluster their recall by associates, rhymes, or both. The

Reprint requests should be sent to J. H. Mueller, Psychology Department, 210 McAlester Hall, University of Missouri, Columbia, Missouri 65201. The author wishes to acknowledge the comments on an earlier draft by Matt Marler and the assistance of Jay Olson in data collection. anxiety-depth hypothesis suggests that high-anxiety subjects would show a deficit in terms of associative organization, as has been found previously, but no deficit should be observed for acoustic organization. As a methodological point, the method of list presentation was either visual or auditory, to determine whether this had any effect on the perception of the presence of the acoustic relationships.

\section{METHOD}

\section{Subjects and Design}

Sixty-four students in introductory psychology courses were selected on the basis of test anxiety scores (Sarason, 1972). High-anxiety subjects had scores greater than $19($ mean $=$ 28.1 ), and low-anxiety subjects had scores below 10 (mean $=7.2)$. The basic design was a 2 by 2 factorial for test anxiety (high, low) and input modality (auditory, visual), with eight men and eight women per cell. Females generally performed better than males, but sex did not enter into any interactions and will not be considered further.

\section{Materials}

The list was composed of 32 words. There were eight base words (e.g., CHAIR), each of which generated a rhyme (SHARE) and an associate (TABLE). These triads were presented in blocked fashion, so that both bases of organization would be as transparent as possible. The order for half the triads on a trial was associate-base-rhyme, then rhyme-base-associate for the rest; order was systematically balanced over trials for each triad. Each triad was separated from the next by an unrelated filler word. With two exceptions, all 32 words were A or AA in ThorndikeLorge frequency; all were uniquely defined by their first two letters and were nonhomophonic. Four different orders of presentation were used.

\section{Procedure}

Each subject had five trials on the list, with a 90-sec written test immediately following each presentation. The instructions emphasized the option to recall in any order. The list was presented to small groups of subjects either by a slide projector or a tape recorder, at a $2-\mathrm{sec}$ rate. The fifth test was followed by a series of digit-span tests from the WAIS requiring about $5 \mathrm{~min}$. A single final test was then given, without further study, as a test of the durability of shallow encodings.

The State Anxiety Inventory (Spielberger, Gorsuch, \& Lushene, 1970) was also administered. Ten of its 20 items were given prior to Trial 1 and the remaining 10 after Trial 5, counterbalancing specific halves as first and last. Analysis indicated that subjects selected on test anxiety indeed differed 
significantly in terms of situational anxiety. High-test anxious subjects had a mean total state anxiety score of $43.5(\mathrm{SD}=8.5)$, and low-test anxious subjects had a mean of $36.3(\mathrm{SD}=6.4)$.

\section{RESULTS $^{1}$}

\section{Immediate Tests}

Recall. Table 1 summarizes performance on Trials 1-5. Low-anxiety subjects recalled more base words $[\mathrm{F}(1,56)$ $=11.86$, $\mathrm{MSe}=2.04]$, more rhymes $[\mathrm{F}(1,56)=11.46$, $\mathrm{MSe}=4.47]$, and more associates $[\mathrm{F}(1,56)=11.50$, MSe $=2.35]$, but their advantage was not significant for the unrelated filler words $[F(1,56)=2.65, \mathrm{p}<.11$, MSe $=3.96]$. The input modality main effect was nonsignificant in each case [though it approached significance for the unrelated filler words, $F(1,56)=$ $3.03, \mathrm{p}<.09, \mathrm{MSe}=3.96$ ] , and there were no Anxiety by Modality interactions for recall of any item type (Fs < 1.38).

For rhyme recall, the Anxiety by Trials interaction revealed that there was no anxiety difference on Trial 1 , but low-anxiety subjects recalled more rhymes thereafter $[\mathrm{F}(4,224)=2.55, \mathrm{MSe}=1.10]$. For total recall, the Modality by Trials interaction indicated that there was less recall on Trial 1 with auditory presentation, but comparable recall resulted for visual and auditory input on Trials $2.5[\mathrm{~F}(4,224)=2.42, \mathrm{MSe}=4.04]$. Trials entered into no other interactions with anxiety or modality, alone or together (Fs $<2.18)$.

Clustering. The pairwise occurrence of a base word and its associate consecutively in output was scored as an associative (deep) cluster, and the consecutive occurrence of the rhyme and base word was scored as an acoustic (shallow) cluster. Low-anxiety subjects exhibited a greater number of clusters of each type $[\mathrm{Fs}(1,56)=10.06$ and 4.12, MSe $=3.56$ and 6.99, for associates and rhymes, respectively]. Neither the modality main effects nor the Anxiety by Modality interactions were significant $(\mathrm{Fs}<1.08)$.

The Anxiety by Mode by Trials interaction approached significance for associative clusters $[\mathrm{F}(4,224)$

Table 1

Average Recall, Clusters, and Whole Triads Recalled per Trial on Trials 1-5 by Test Anxiety and Presentation Modality

\begin{tabular}{lrrrrr}
\hline & \multicolumn{2}{c}{ High Anxiety } & & \multicolumn{2}{c}{ Low Anxiety } \\
\cline { 2 - 3 } \cline { 5 - 6 } \cline { 5 - 6 } & Auditory & Visual & & Auditory & Visual \\
\hline Recall & & & & & \\
$\quad$ Associate & 5.61 & 5.58 & & 6.03 & 6.33 \\
Rhyme & 4.05 & 4.43 & & 4.91 & 5.16 \\
Base & 5.80 & 5.69 & & 6.16 & 6.43 \\
Unrelated & 3.56 & 3.84 & & 3.81 & 4.31 \\
Total & 19.03 & 19.53 & & 20.91 & 22.23 \\
Clusters & & & & \\
$\quad$ Associates & 4.46 & 4.51 & & 4.96 & 5.35 \\
Rhymes & 2.30 & 2.01 & & 2.81 & 2.70 \\
$\quad$ Triads & & & & & \\
Consecutive & .36 & .32 & & .45 & .43 \\
$\quad$ Anywhere & .57 & .58 & .67 & .68 \\
\hline
\end{tabular}

$=2.38, \mathrm{p}<.06, \mathrm{MSe}=1.67]$. With auditory input, the low-anxiety advantage was nonexistent on early trials, but developed from Trial 3 on; with visual input, the low-anxiety advantage was greatest on the early trials, with low- and high-anxiety subjects converging thereafter. Trials did not enter into any other interactions with anxiety or modality, alone or together $(\mathrm{Fs}<1.06)$.

Triad recall. An alternative index of organization would be the number of times an entire triad was recalled: base word, associate, and rhyme, either consecutively (in any order) or anywhere in output. A minimum requirement would seem to be the recall of the base word, so that it could be used to access the rhyme and/or associate. Thus, the number of whole triads recalled (by each criterion) was divided by the number of base words recalled (i.e., opportunities to recall a whole triad), and Table 1 presents the probability of recalling whole triads by the strict (consecutive) and lenient (anywhere) criteria. [The lenient criterion is of interest, since with a written test subjects can reinspect their output record as time permits; thus, on reviewing, subjects may retrieve the initially missed member(s) of a triad, but then must write it (them) in a nonconsecutive location.]

Low-anxiety subjects displayed more organization of this type for both triads consecutively and anywhere $[\mathrm{Fs}(1,56)=4.68$ and $9.96, \mathrm{MSe}=.17$ and .09 , respectively]. There were no differences due to input modality, nor Anxiety by Modality interactions $($ Fs $<1)$. As was also the case for recall and clustering, of course, these behaviors increased significantly over trials, but trials entered into only one interaction with anxiety or modality: For wholes anywhere, the Anxiety by Trials interaction indicated that the anxiety subgroups did not differ on Trial 1, but with practice the low-anxiety subjects increased their usage of this organizational behavior to a greater extent than high-anxiety subjects $[\mathrm{F}(4,224)=2.64, \mathrm{MSe}=.03]$.

\section{Delayed Test}

The results of the delayed test can be summarized briefly: There were no anxiety or modality main effects or Anxiety by Modality interactions for recall, clustering, or triad recall (all ps $>.05$ ). Low-anxiety subjects continued to perform slightly better, but not significantly so. Recall and clustering of associates continued to exceed that of rhymes by about the same proportion as on Trials 1-5; that is, rhyme performance did not decline markedly. Over all groups, mean recall was 7.14 and 6.61, and clustering means were 5.97 and 3.43 , for associates and rhymes, respectively.

\section{Digit Span}

The digit-span tests revealed superior performance by low-anxiety subjects in both the forward and backward directions $[\mathrm{Fs}(1,56)=6.19$ and $4.86, \quad \mathrm{MSe}=1.58$ and 2.35, respectively]. Mean forward spans were 7.1 and 6.3, and mean backward spans were 5.7 and 4.8 , for low- and high-anxiety subjects, respectively. 


\section{DISCUSSION}

These results corroborate earlier findings of a deficit in free recall performance for high-anxiety subjects. This deficit was quite general, occurring for all types of items in recall, for both associative and acoustic clustering, and for triad recall. On the surface, this would appear to contradict the hypothesis that the high-anxiety subjects' deficit is limited to semantic content. High-anxiety subjects did show a reduced deficit for acoustic organization, but the difference was still significant. If rhyme clusters in recall can actually be considered as involving shallowly processed attributes, then the fact that high-anxiety subjects displayed a deficit for rhymes as well as associates constitutes a problem for the anxiety-depth analysis.

There are some problems which must be resolved before a final decision can be made. Primarily, it is necessary that the alternative bases for organization be equally potent and obvious to the subjects. While the blocked presentation method was used to alleviate problems in discovering the rhyming relationships, there was still less organization by rhymes than associates (as has been shown before, e.g., Dolinsky, 1972). This suggests that the rhyme basis still was not as potent, as obvious, or as useful as the associative relationships. Consequently, it is quite possible that the acoustic relationships may have required more processing to discover or use than would be implied in describing them as involving superficially encoded physical-shallow features, with the result that the high-anxiety subjects would still be expected to be at a disadvantage. That is, since the highanxiety subjects seem to have some limitations in processing and utilizing semantic information and/or weak cues, a physical basis for organization that requires considerable processing does not constitute a reasonable test. An adequate test of the anxiety-depth hypothesis requires alternative bases for organization which are of equal salience but different depth. Such ideal lists may be quite difficult to construct, if they are in fact possible at all.

\section{REFERENCES}

Broadbent, D. E. Decision and stress. London: Academic Press, 1971.

CRAIK, F. I. M., \& LockhaRT, R. S. Levels of processing: A framework for memory research. Journal of Verbal Learning and Verbal Behavior, 1972, 11, 671-684.

Dolinsky, R. Clustering and free recall with alternative organizational cues. Journal of Experimental Psychology, 1972, 95, 159-163.

Mueller, J. H. Anxiety and cue utilization in human learning and memory. In M. Zuckerman \& C. D. Spielberger (Eds.), Emotions and anxiety: New concepts. methods and applications. Potomac, Md: L. Erlbaum, 1976.

SARASON, I. G. Experimental approaches to test anxiety: Attention and uses of information. In C. D. Spielberger (Ed.), Anxiety: Current trends in theory and research (Vol. 2). New York: Academic Press, 1972.

SchwarTz, S. Individual differences in cognition: Some relationships between personality and memory. Journal of Research in Personality, 1975, 9, 217-225.

Spielberger, C. D., Gorsuch, R. L., \& Lushene, R. E. Manual for the state-trait anxiety inventory. Palo Alto, Calif: Consulting Psychologists Press, 1970.

\section{NOTE}

1. Effects described as significant involve at least $p<.05$, unless noted otherwise.

(Received for publication August 30, 1976.) 